\title{
Increased Expression of Neuregulin 1 and erbB2 Tyrosine Kinase in the Bladder of Rats With Cyclophosphamide-Induced Interstitial Cystitis
}

\author{
Ki Hak Song ${ }^{1}$, Chang Shik Youn², Chung Lyul Lee ${ }^{1}$, Seung Woo Yang ${ }^{1}$, Young Seop Chang ${ }^{3}$, Seoung Woo Jeong ${ }^{4}$, \\ Chong Koo Sul ${ }^{1}$ \\ ${ }^{1}$ Department of Urology, Chungnam National University School of Medicine, Daejeon, Korea \\ ${ }^{2}$ Department of Urology, The Catholic University of Korea, Daejeon St. Mary's Hospital, Daejeon, Korea \\ ${ }^{3}$ Department of Urology, Konyang University College of Medicine, Daejeon, Korea \\ ${ }^{4}$ Department of Physiology, Yonsei University Wonju College of Medicine, Wonju, Korea
}

Purpose: The aim of this study was to evaluate changes in expressions of neuregulin (NRG) 1 and erbB2 tyrosine kinase (ErbB2) in bladders of rats with cyclophosphamide (CYP)-induced interstitial cystitis (IC).

Methods: Twenty-four Sprague-Dawley rats were divided into the IC group $(n=16)$ and the control group $(n=8)$. After inducing IC with intraperitoneal CYP injection, expressions of NRG1 and ErbB2 were analyzed using western blotting and reverse transcriptase-polymerase chain reaction.

Results: In Western blotting, relative intensities and distributions of both NRG1 and ErbB2 were approximately 1.5- and 3.2fold higher, respectively, in the IC group than in the control group (mean \pm standard deviation: $1.42 \pm 0.09$ vs. $0.93 \pm 0.15$ and $0.93 \pm 0.16$ vs. $0.29 \pm 0.08, P<0.05$ ). In the rat bladder samples, mRNA expression levels of NRG1 and ErbB2 were higher in the IC group than in the control group $(\mathrm{P}<0.05)$.

Conclusions: Our study has demonstrated significant changes in mRNA expression and immunoreactivity of NRG1 and ErbB2 receptors in the urinary bladder after CYP-induced IC. These results suggest that the up-regulated NRG1 may play a role in inducing an overactive bladder and promoting regeneration in the inflammatory bladder with CYP-induced IC.

Keywords: Neuregulin-1; Receptor, ErbB-2; Cystitis, Interstitial; Rats

- Fund Support: This study was financially supported by an educational fund (No. 2009-1650) of Chungnam National University, Daejeon,

Korea.

- Research Ethics: All animal experiments followed a protocol approved by the Ethics Committee on animal research at Chungnam National

University (Daejeon, Korea).

- Conflict of Interest: No potential conflict of interest relevant to this article was reported.

\section{INTRODUCTION}

Patients with interstitial cystitis (IC) or painful bladder syndrome (PBS) exhibit urinary urgency, frequency, and suprapubic and pelvic pain related to bladder filling [1,2]. IC is on the increase and is a frequently observed urologic disease [3]. Bladder inflammation, urethra stimulus in the lower urinary tract, mechanical bulging within the uterus or vagina, and urethral occlu-

Corresponding author: Chong Koo Sul (i) http://orcid.org/0000-0002-7225-1496 Department of Urology, Chungnam National University Hospital, Chungnam National University School of Medicine, 282 Munhwa-ro, Jung-gu, Daejeon 35015, Korea

E-mail: snowman@cnu.ac.kr / Tel: +82-42-280-7776 / Fax: +82-42-257-0966 Submitted: June 11, 2015 / Accepted after revision: July 27, 2015 
sion have been used in animal models of urological conditions such as IC, overactive bladder syndrome, and detrusor hyperactivity $[4,5]$. It has been suggested that IC/PBS is a type of hypersensitivity disorder that affects the bladder and other somatic/ visceral organs induced by neural up-regulation [6] with many overlapping symptoms and pathophysiology $[7,8]$.

Neuregulins (NRGs) are pleiotropic growth factors of the epidermal growth factor family. Their roles in neuronal and glial development have been investigated extensively [9]. We reported that NRG1 up-regulated the expression of nicotinic acetylcholine receptors via the overexpression of the erbB2 tyrosine kinase (ErbB2) receptor by using a mitogen-activated protein kinase (MAPK) cascade in neurons [10]. However, the expression and role of NRG1 in cyclophosphamide (CYP)-induced IC has not been studied in depth. The purpose of our study is to evaluate changes in expressions of NRG1 and ErbB2 in the bladder of a CYP-induced IC rat model.

\section{MATERIALS AND METHODS}

\section{Experimental Animals and Induced IC}

The experimental animals used in this study were female Sprague-Dawley rats (200-250 g, Daehan Biolink Co., Daejeon, Korea), and all animal experiments followed a protocol that was approved by the Ethics Committee on animal research at Chungnam National University, Daejeon, Korea. The animals were raised in pairs in a cage covered with sawdust. Water and feed were provided ad libitum at standard laboratory conditions $\left(25^{\circ} \mathrm{C} \pm 1^{\circ} \mathrm{C}, 55 \% \pm 5 \%\right.$ humidity, 12 -hour alternating light-dark cycle). In total, 24 rats were used in this study and they were randomly divided into two groups (16 in the experimental group and 8 in the control group). The rats in the experimental group were intraperitoneally injected with $300 \mathrm{mg} / \mathrm{kg}$ of CYP (Sigma-Aldrich Co., St. Louis, MO, USA) at a volume of 10 $\mathrm{mL} / \mathrm{kg}$, and those in the control group were injected with physiological saline by using previously reported methods [2].

\section{RNA Extraction and Construction of CDNA}

Cystectomy was performed on the seventh day after the initial administration of CYP. Total RNA was extracted from the bladder tissue of control and CYP-treated rats. One milliliter of TRIzol (Invitrogen, Carlsbad, CA, USA) was added to the bladder tissue and homogenized in a 5-mL glass tube. The homogenate was transferred to a $1-\mathrm{mL}$ tube and mixed with $200 \mu \mathrm{L}$ of chloroform. After incubation for 5 minutes at $4^{\circ} \mathrm{C}$, the homog- enate was centrifuged for 10 minutes at $13,200 \times \mathrm{g}$ at $4^{\circ} \mathrm{C}$. The supernatant was transferred to a clean tube and $1,000 \mu \mathrm{L}$ of isopropyl alcohol (Sigma-Aldrich Co.) was added, followed by incubation for 30 minutes at $4^{\circ} \mathrm{C}$. The tube was then centrifuged for 8 minutes at $13,200 \times \mathrm{g}$ at $4^{\circ} \mathrm{C}$. Then, the supernatant was discarded, pellet was mixed with $500 \mu \mathrm{L}$ of $75 \%$ ethanol, and centrifuged for 10 minutes at $13,200 \times \mathrm{g}$ at $4^{\circ} \mathrm{C}$. After the supernatant was discarded, the pellet was dried at room air for 5 minutes, dissolved with diethylpyrocarbonate (DEPC, SigmaAldrich Co.)-treated water, and stored at $-75^{\circ} \mathrm{C}$. The quality and integrity of the RNA were confirmed by agarose gel electrophoresis and ethidium bromide staining, followed by visual examination under ultraviolet light. cDNA was then prepared from $1 \mu \mathrm{g}$ of random primers using a First Strand cDNA Synthesis Kit (TaKaRa, Tokyo, Japan) according to the manufacturer's protocol.

\section{Reverse Transcriptase-Polymerase Chain Reaction}

Total RNA was isolated from CYP-induced cystitis bladder tissues and normal bladder tissues in rats by using the TRIzol reagent according to the manufacturer's instructions. cDNA was synthesized using First Strand cDNA Synthesis Kit (Fermentas Inc., Glen Burnie, MD, USA) with oligo-dT as primers. After an initial denaturation step at $94^{\circ} \mathrm{C}$ for 2 minutes, 35 cycles of amplification were performed at $57^{\circ} \mathrm{C}$ for 60 seconds, followed by 25 minutes at $57^{\circ} \mathrm{C}$ as an additional extension step in reverse transcriptase-polymerase chain reaction (RT-PCR). PCR products were analyzed by electrophoresis on 1.5\% agarose gels. Glyceraldehyde-3-phosphate dehydrogenase (GAPDH) was used as a housekeeping gene. The primer sequences were as follows: for NRG1 (358-bp), sense 5'-CCCGCC-GGCTATTGGTGACTT- ${ }^{\prime}$ ' and antisense 5'-ATGACCACCCCGGCTCGTATGT-3'; for ErbB2 (483-bp), sense $5^{\prime}$-AGCCCCCAGCCC-GAGTATGTGAAC- ${ }^{\prime}$ ' and antisense 5'-GAGCCGTCTGCCCTGGAT-GTAATG-3'; for GAPDH (189-bp), sense 5'-GATGGCATGGACTGTGGTCA-3' and antisense 5'-GTCATCATCTCCGCCCCTTC-3'.

\section{Western Blotting}

Rat bladder tissues were separated by $12 \%$ sodium dodecyl sulfate-polyacrylamide gel electrophoresis and the proteins were then transferred to a polyvinylidene fluoride membrane (Dyneon, 3M, St. Paul, MN, USA). The blots were then washed with Tris-buffered saline Tween-20 (10 mM Tris-HCl, pH 7.6, 150 $\mathrm{mM} \mathrm{NaCl}, 0.05 \%$ Tween-20). The membrane was blocked with $5 \%$ skimmed milk for 1 hour and incubated with the appropriate 
primary antibody. Polyclonal rabbit antibodies for ErbB2 (1:200, Abcam, Cambridge, MA, USA) and monoclonal mouse antibodies for NRG1 (1:200, Abcam) were used. The membrane was then washed and the antibody reactions were detected by goat antimouse antibodies for NRG1 and goat antirabbit antibodies for ErbB2 and $\beta$-actin (1:200, Abcam) conjugated to horseradish peroxidase. The antibody incubations were performed in an incubator at $4^{\circ} \mathrm{C}$. The bands were visualized using enhanced che-
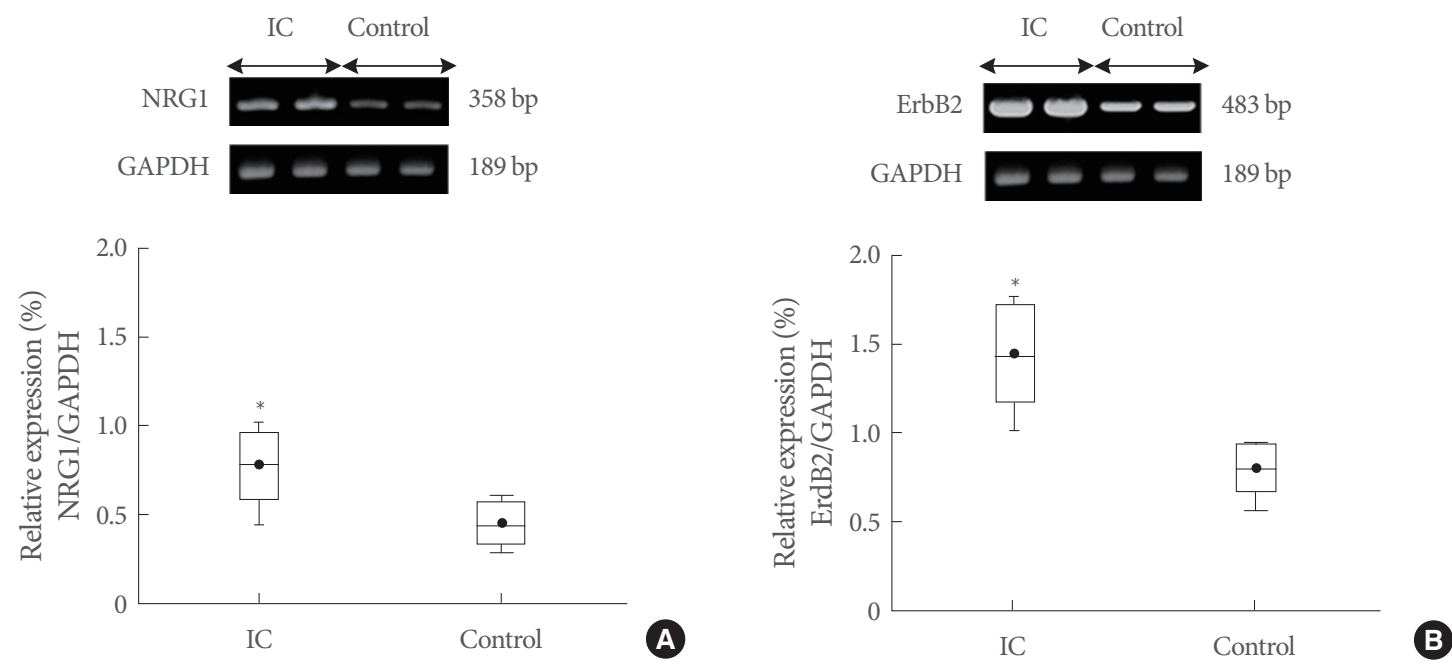

Fig. 1. Representative mRNA expression and relative distribution of NRG1 (A) and ErbB2 (B) in interstitial cystitis (IC) and control groups. GAPDH was used as the loading control. Values of NRG1 and ErbB2 expression are normalized relative to GAPDH expression. The asterisk indicates a significant difference $(\mathrm{P}<0.05)$. NRG, neuregulin; ErbB2, erbB2 tyrosine kinase; GAPDH, glyceraldehyde-3-phosphate dehydrogenase.
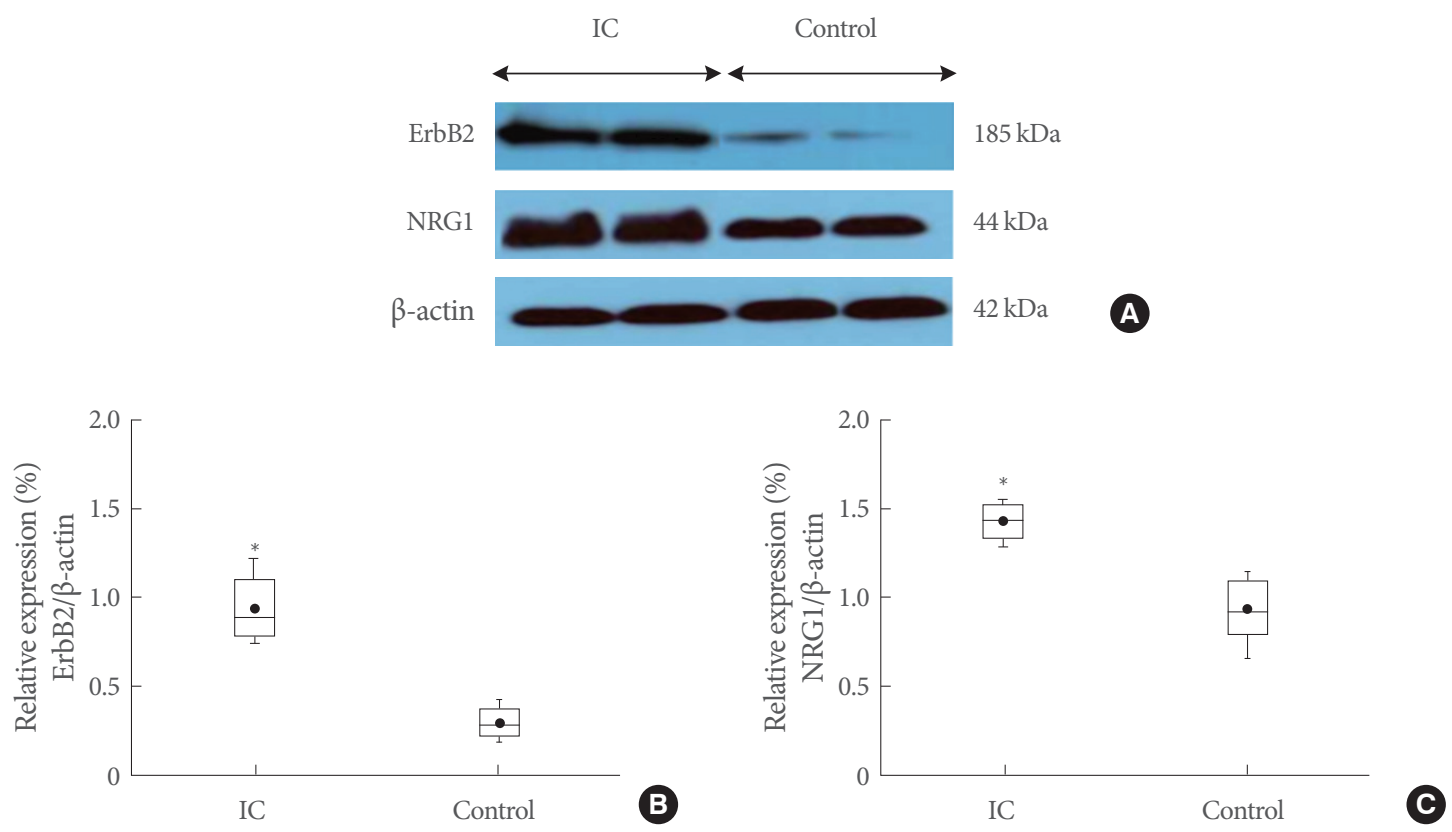

Fig. 2. Representative immunoblots (A) and relative distribution of ErbB2 (B) and NRG1 (C) protein in interstitial cystitis (IC) and control groups. $\beta$-actin was used as the loading control. Values of NRG1 and ErbB2 expression are normalized relative to $\beta$-actin expression. The asterisk indicates a significant difference (Mann-Whitney test; $\mathrm{P}<0.05)$. ErbB2, erbB2 tyrosine kinase; NRG, neuregulin. 
miluminescence (Vilber Lourmat, Marne La Vallée, France).

\section{Statistical Analysis}

All data were presented as mean \pm standard deviation. For comparison between two groups, the Mann-Whitney test was used. All calculations were performed using IBM SPSS Statistics ver. 20.0 (IBM Co., Armonk, NY, USA). Differences of $\mathrm{P}<0.05$ were considered statistically significant.

\section{RESULTS}

\section{mRNA Expression of NRG1 and ErbB2}

We examined the mRNA expression levels of NRG1 and ErbB2 in bladder tissues from the CYP-induced cystitis and control groups using RT-PCR. The mRNA expression level of NRG1 was higher in bladder samples of rats with CYP-induced IC than in those of control rats (Fig. 1A) $(\mathrm{P}<0.05)$. The mRNA expression of ErbB2 yielded similar results to that of NRG1. As a whole, CYP-induced IC showed significantly higher levels of ErbB2 (Fig. 1B) $(\mathrm{P}<0.05)$. Gene expression was normalized to the expression of GAPDH.

\section{Western Blot Assays of NRG1 and ErbB2}

We examined the expression of NRG1 (44 kDa) and ErbB2 $(185 \mathrm{kDa})$ by immunoblotting analysis in bladder tissues of CYP-induced IC and control rats (Fig. 2A). The relative intensities and distributions of both NRG1 and ErbB2 were approximately 1.5- and 3.2-fold higher, respectively, in the IC group than in the control group $(1.42 \pm 0.09$ vs. $0.93 \pm 0.15$ and 0.93 \pm 0.16 vs. $0.29 \pm 0.08, \mathrm{P}<0.05$ ) (Fig. $2 \mathrm{~B}, \mathrm{C}$ ).

\section{DISCUSSION}

In recent years, IC/PBS has been defined by the Society of Urodynamics, Female Pelvic Medicine \& Urogenital Reconstruction as an unpleasant sensation (pain, pressure, and discomfort) perceived to be related to the urinary bladder, associated with lower urinary tract symptoms of more than six weeks in duration, in the absence of infections or other identifiable causes [11]. When patients experience IC/PBS, it may be difficult to identify whether they have a primary bladder disorder or whether the symptoms are secondary phenomena resulting from another cause unrelated to the bladder. For these reasons, it is difficult to diagnose and treat IC/PBS. Furthermore, cystometric findings by using $0.3 \mathrm{M} \mathrm{KCl}$ did not help in differentiat- ing IC from detrusor overactivity [12]. Therefore, IC/PBS may be regarded as a part of the continuum of painful versus nonpainful overactive bladder syndromes [12,13] and hypersensitivity disorders that affect the bladder and other somatic/visceral organs induced by neural up-regulation [6].

NRGs have been investigated extensively with regard to their roles in neuronal and glial development [9]. Vascular endothelial cells express ErbB receptors and their ligand, NRG1, and can respond to vascular preservation and angiogenic responses of the endothelium and cardiac smooth muscle cells in the heart via the NRG/ErbB pathway [14]. In this study, we demonstrated increased protein and mRNA expression of NRG1 and ErbB2 in the IC group compared with the control group. The results may reflect interactions among the urothelium with an overexpression of NRG1 and ErbB2, inflammatory infiltrates, interstitial cells, and myofibroblasts. It was hypothesized that NRG1 might have an important role in detrusor overactivity by directly responding at sensory nerve endings or indirectly increasing the expression of sensory neuropeptides. Calvo et al. [15] reported an increase in NRG1 within the dorsal horn and observed the activation of ErbB2 specifically within the microglia after spinal nerve ligation. NRG1/ErbB represents a signaling pathway that regulates the injury response. The blockage of ErbB2 receptors or sequestration of endogenous NRG reduced mechanical pain-related hypersensitivity and cold allodynia. Altered visceral sensations from the urinary bladder (i.e., pain at low or moderate bladder filling) that accompany IC or PBS may be mediated by many factors, including changes in the properties of the peripheral bladder afferent pathways, such that bladder afferent neurons respond in an exaggerated manner to normally innocuous stimuli. These changes may be mediated, in part, by inflammatory changes in the urinary bladder. Among potential mediators of inflammation, neurotrophins and proinflammatory cytokines have been implicated in the sensitization process $[16,17]$. Moreover, we suggested that the overexpression of NRG1 in IC/PBS leads to the activation of extracellular signal-regulated kinase (ERK) pathways, followed by the activation of phosphatidylinositol-3-kinase by using the ErbB pathway [10]. Cruz and Cruz [18] reported that the activation of ERK might depend on the expression of pronociceptive enzymes and cytokines including inducible nitric oxide synthase, cyclooxygenase-2 (COX-2), interleukin-1 beta (IL$1 \beta)$, tumor necrosis factor alpha (TNF- $\alpha$ ), and interleukin-6 (IL-6), making the ERK pathway a key signaling pathway in glial cells. We have previously reported an increased expression 
of nitric oxide synthase and histologic changes in the bladder of rats with CYP-induced IC [2].

ERK is a member of the MAPK family, which consists of the classical cascades p38, c-Jun N-terminal kinases (JNK), and ERK 1 and $2[19,20]$. MAPK is an important signaling pathway characterized by a central motif of three kinases and is associated with cell proliferation [21], differentiation, development, inflammation [22], and apoptosis [23,24]. The activation of MAPK leads to different cellular outcomes. In general, the ERK pathway responds to growth factor signals, whereas p38 and JNK pathways respond to inflammation and apoptosis [20]. Various membrane-bound receptors, including growth factor receptors, $G$ protein-coupled receptors, estrogen receptors, and ionic channels are involved in the activation of the ERK pathway [18]. Corrow and Vizzard [25] reported that the activation of ERK pathways was significantly up-regulated in the urothelium after 48 hours or chronic CYP treatment. They suggested that IC therapies targeted at the activation of the ERK pathway might improve urinary bladder function. Recent reports have shown that the inhibition of ErbB2 receptors disrupted cardiomyocyte proliferation in response to injury, whereas myocardial NRG1 overexpression enhanced this proliferation in zebrafish [26]. Furthermore, D'Uva et al. [27] reported that ErbB2 is both necessary for cardiomyocyte proliferation and sufficient to reactivate postnatal cardiomyocyte proliferative and regenerative potentials following myocardial infarction. Therefore, NRG1 may play a role in proliferation and regeneration on the urothelium and detrusor muscle with CYP-induced IC. Furthermore, it may indirectly modulate nociceptive signaling by altering the expression of pronociceptive enzymes and cytokines that are thought to play a central role in inflammation or tissue injuryinduced pain and hypersensitivity.

In conclusion, our findings indicate significant changes in the mRNA expression and immunoreactivity of NRG1 and ErbB2 receptors in the urinary bladder with CYP-induced IC. These results suggest that the up-regulated NRG1 may play a role in inducing an overactive bladder and promoting regeneration in the inflammatory bladder with CYP-induced IC via the activation of the ERK-MAPK pathway.

\section{REFERENCES}

1. Hanno PM, Erickson D, Moldwin R, Faraday MM; American Urological Association. Diagnosis and treatment of interstitial cystitis/ bladder pain syndrome: AUA guideline amendment. J Urol 2015;
193:1545-53.

2. Cho KH, Hyun JH, Chang YS, Na YG, Shin JH, Song KH. Expression of nitric oxide synthase and aquaporin-3 in cyclophosphamide treated rat bladder. Int Neurourol J 2010;14:149-56.

3. Davis NF, Brady CM, Creagh T. Interstitial cystitis/painful bladder syndrome: epidemiology, pathophysiology and evidence-based treatment options. Eur J Obstet Gynecol Reprod Biol 2014;175:307.

4. McMahon SB, Abel C. A model for the study of visceral pain states: chronic inflammation of the chronic decerebrate rat urinary bladder by irritant chemicals. Pain 1987;28:109-27.

5. Lantéri-Minet M, Bon K, de Pommery J, Michiels JF, Menetrey D. Cyclophosphamide cystitis as a model of visceral pain in rats: model elaboration and spinal structures involved as revealed by the expression of c-Fos and Krox-24 proteins. Exp Brain Res 1995;105:220-32.

6. Nazif O, Teichman JM, Gebhart GF. Neural upregulation in interstitial cystitis. Urology 2007;69(4 Suppl):24-33.

7. Bade J, Ishizuka O, Yoshida M. Future research needs for the definition/diagnosis of interstitial cystitis. Int J Urol 2003;10 Suppl:S31-4.

8. Payne CK, Terai A, Komatsu K. Research criteria versus clinical criteria for interstitial cystitis. Int J Urol 2003;10 Suppl:S7-S10.

9. Mei L, Xiong WC. Neuregulin 1 in neural development, synaptic plasticity and schizophrenia. Nat Rev Neurosci 2008;9:437-52.

10. Kim HG, Lee CK, Cho SM, Whang K, Cha BH, Shin JH, et al. Neuregulin 1 up-regulates the expression of nicotinic acetylcholine receptors through the ErbB2/ErbB3-PI3K-MAPK signaling cascade in adult autonomic ganglion neurons. J Neurochem 2013;124:502-13.

11. Hanno P, Dmochowski R. Status of international consensus on interstitial cystitis/bladder pain syndrome/painful bladder syndrome: 2008 snapshot. Neurourol Urodyn 2009;28:274-86.

12. Philip J, Willmott $S$, Irwin P. Interstitial cystitis versus detrusor overactivity: a comparative, randomized, controlled study of cystometry using saline and 0.3 M potassium chloride. J Urol 2006;175:566-70.

13. Homma Y, Ueda T, Tomoe H, Lin AT, Kuo HC, Lee MH, et al. Clinical guidelines for interstitial cystitis and hypersensitive bladder syndrome. Int $J$ Urol 2009;16:597-615.

14. Hedhli N, Kalinowski A, S Russell K. Cardiovascular effects of neuregulin-1/ErbB signaling: role in vascular signaling and angiogenesis. Curr Pharm Des 2014;20:4899-905.

15. Calvo M, Zhu N, Tsantoulas C, Ma Z, Grist J, Loeb JA, et al. Neuregulin-ErbB signaling promotes microglial proliferation and chemotaxis contributing to microgliosis and pain after peripheral nerve injury. J Neurosci 2010;30:5437-50.

16. Jiang YH, Peng CH, Liu HT, Kuo HC. Increased pro-inflammatory cytokines, C-reactive protein and nerve growth factor expressions 
in serum of patients with interstitial cystitis/bladder pain syndrome. PLoS One 2013;8:e76779.

17. Dray A. Inflammatory mediators of pain. Br J Anaesth 1995;75:12531.

18. Cruz CD, Cruz F. The ERK 1 and 2 pathway in the nervous system: from basic aspects to possible clinical applications in pain and visceral dysfunction. Curr Neuropharmacol 2007;5:244-52.

19. Imajo M, Tsuchiya Y, Nishida E. Regulatory mechanisms and functions of MAP kinase signaling pathways. IUBMB Life 2006;58:3127.

20. Yang SH, Sharrocks AD, Whitmarsh AJ. MAP kinase signalling cascades and transcriptional regulation. Gene 2013;513:1-13.

21. Chan LK, Chiu YT, Sze KM, Ng IO. Tensin4 is up-regulated by EGF-induced ERK1/2 activity and promotes cell proliferation and migration in hepatocellular carcinoma. Oncotarget 2015;6:2096476.

22. Dugan C, Malley S, Arms L, May V, Vizzard MA. Role of c-Jun Nterminal kinase (JNK) activation in micturition reflexes in cyclophosphamide (CYP)-induced cystitis in female rats. J Mol Neurosci 2014;54:360-9.
23. Jie P, Hong Z, Tian Y, Li Y, Lin L, Zhou L, et al. Activation of transient receptor potential vanilloid 4 induces apoptosis in hippocampus through downregulating PI3K/Akt and upregulating p38 MAPK signaling pathways. Cell Death Dis 2015;6:e1775.

24. Swiatkowski S, Seifert HH, Steinhoff C, Prior A, Thievessen I, Schliess F, et al. Activities of MAP-kinase pathways in normal uroepithelial cells and urothelial carcinoma cell lines. Exp Cell Res 2003;282:48-57.

25. Corrow KA, Vizzard MA. Phosphorylation of extracellular signalregulated kinases in urinary bladder in rats with cyclophosphamide-induced cystitis. Am J Physiol Regul Integr Comp Physiol 2007;293:R125-34.

26. Gemberling M, Karra R, Dickson AL, Poss KD. Nrg1 is an injuryinduced cardiomyocyte mitogen for the endogenous heart regeneration program in zebrafish. Elife 2015;4. http://dx.doi.org/10.7554/ eLife.05871.

27. D’Uva G, Aharonov A, Lauriola M, Kain D, Yahalom-Ronen Y, Carvalho S, et al. ERBB2 triggers mammalian heart regeneration by promoting cardiomyocyte dedifferentiation and proliferation. Nat Cell Biol 2015;17:627-38. 